\title{
Quality competition in healthcare services with regional regulators: A differential game approach
}

\author{
Michele Bisceglia*, Roberto Cellini ${ }^{\dagger}$ Luca Grilli ${ }^{\ddagger}$
}

February 10, 2018

\begin{abstract}
This article proposes a differential-game model, in order to analyze markets in which regional regulation is operative and competition is based on quality. The case we have in mind is healthcare public service, where consumers (patients) choose the provider mainly basing on the providers' location and the quality of services, while prices play a more limited role. In most European countries, within the same

${ }^{*}$ Dipartimento di Scienze aziendali, economiche e metodi quantitativi, Università degli Studi di Bergamo, Via dei Caniana 2, 24127 Bergamo, Italy, michele.bisceglia@unibg.it.

${ }^{\dagger}$ Dipartimento di Economia e Impresa, Università degli Studi di Catania, Corso Italia 55, 95129 Catania, Italy, cellini@unict.it.

${ }^{\ddagger}$ Dipartimento di Economia, Università degli Studi di Foggia, Largo Papa Giovanni Paolo II, I-71100 Foggia, Italy, luca.grilli@unifg.it.
\end{abstract}


State, regional (or local) providers compete on quality to attract demand. Market regulation is set at national and/or regional level. Our model highlights the features of equilibrium in such a framework, and specifically investigates how the differences in product quality evolve among regions, and how inter-regional demand flows behave. Differently from some available similar models, that do not take into account the regional dimension of the decision process, we find that quality differentials among regions may persist in equilibrium.

Keywords: Healthcare Services; Diagnosis Related Group; Differential Game; Quality Competition; Regional Regulators.

Mathematical Subject Classification: 91A23, 91A25, 49N90, 91A80, 91B15, 91A10.

JEL Classification: C72, C73, H41, I11, I18, P36, L13, R38.

\section{Introduction}

This article flows within the literature stream dealing with dynamic quality competition in markets under price regulation. A reference case is given by healthcare service, where prices are typically regulated, providers compete on quality, and consumers' demand choices are mainly driven by product quality and providers' location. In healthcare markets, the current relevance of quality competition is made clear by the fact that many reforms have implemented over the past years in several countries, with the final aim of increasing the product quality through harsher quality competition among providers. Generally speaking, such reforms allow the final consumers to choose the provider. Given that prices are regulated (and paid by a Public Authority, on a prospective payment system, or by insurance companies), 
the consumers' choices are based on the quality, along with the localization of the providers (Aiura [1]), rather than price. Thus, the providers have to compete to attract demand (and payments), by improving the quality of the offered services. However, our model can be appropriate for other sectors with similar characteristics, like education or long-term care.

A body of literature exists, focusing on the effect of different forms of competition and different regulation mechanisms upon the available quality level over time: Brekke et al. [4], [5], Siciliani et al. [18] are among the main references - a review is provided by Brekke et al. [6]. The specific problems studied in the mentioned articles range from the dynamic evolution of investment efforts made by providers and the corresponding available quality levels, to the relation between regulated prices and investment efforts to improve quality. Attention is paid to how different assumptions concerning the information sets used by players, and the corresponding game solution concepts, affect the features of the equilibrium path of control and state variables, and steady state allocation.

However, in this literature vein, the fact that different providers have a regional character, and regulation of relevant variables is made at the regional level, is usually overlooked. In Bisceglia et al. [3] spatial competition, both intra-regional and inter-regional, between providers is studied by means of a sequential game.

In the present article we aim to merge the mentioned research points concerning quality competition with a different research point, specifically 
concerning the regional distribution of the service quality, the regional regulation, and the effect of decentralization decision processes upon available quality: the reference literature body -limiting our attention to markets with regulated prices and quality competition- includes, e.g., Balia et al. [2], Brekke et al. [7], [8], Levaggi et al. [16], [17], along with Bisceglia et al. $[3]$.

While the former literature line employs dynamic -more specifically, differential- game tools to analyze the evolution of quality over time, the latter line typically resorts to static -or, at most, repeated or sequentialgames. In the present article we propose a differential game model with the main aim of analyzing the implications of decentralized decision processes -specifically, regional regulation- upon the available quality levels. The model can be interpreted as a combination between models belonging to the mentioned literature lines. However, its conclusions are far from being a trivial sum of the points made by available articles. Available differentialgame models generally consider symmetric situations, and fail in highlighting permanent quality differentials across providers; on the other hand, static models with asymmetry between providers can not analyze how persistent over time asymmetries are. Our present model shows that an equilibrium exists with different levels of quality across regions; differences in quality are permanent, but they may shrink or enlarge over time, also depending on the decision rules taken by the regulators. The model we present is analytically solvable thanks to its linear-quadratic structure; it provides some insights 
on the problems faced by providers and regional regulators in markets with quality competition and price regulation.

The value added of the present model, as compared to available theoretical models, rests in the analysis of the evolution of quality across regions and in showing that permanent differences may persist, though in the presence of a large degree of symmetry in parameter configuration across regions: We show here that an equilibrium exists with different levels of quality across regions; differences in quality are permanent, but they may shrink or enlarge over time, also depending on the decision rules taken by the regulators.

The structure of the article is as follows. Section 2 presents the model set-up. Section 3 presents, discusses and compares the open-loop and the feedback closed-loop solution of the game, assuming that regional regulators choose the investments able to move the quality. Section 4 presents a modification of the model, assuming that investments in quality are set by the providers, that are interested in profit rather than social welfare. Section 5 mentions further possible modifications, and concludes.

\section{The model set-up}

The basic set-up of the model is common to a number of available articles based on the Hotelling linear city framework (Hotelling [14]; see Calem and Rizzo [10], as a seminal contribution for the literature relevant to this present analysis; see also Brekke et al. [4]). 
Consider a market with two providers located at either end of the unit line $S=[0,1]$. On this line segment there is a uniform distribution of consumers, with total mass equal to 1 . Since our reference example is healthcare provision, we will even refer to hospitals and patients for providers and consumers, respectively. Assuming that each consumer inelastically demands one unit of the considered service, the utility of a consumer located at $x \in S$ and buying from provider $i$, located at $z_{i} \in\{0,1\}$, is given by

$$
U\left(x, z_{i}\right)=v+k q_{i}-\tau\left|x-z_{i}\right|,
$$

where $v>0$ is a parameter representing the gross valuation of consumption, $q_{i}$ is the quality of the product (service) offered by provider $i, k>0$ is a parameter measuring the marginal willingness to pay for quality, and $\tau>0$ is the marginal transportation cost. In what follows we set $k=1$ without loss of generality. Notice that in the case of competition among hospitals, it makes sense to assume that physical locations are fixed; the fact that locations are at the end points of the line is immaterial to our conclusions. Since the distance between providers is equal to one, the consumer who is indifferent between $i$ and $j$ is located at $x_{i}^{D}$, is characterised by

$$
v-\tau x_{i}^{D}+q_{i}=v-\tau\left(1-x_{i}^{D}\right)+q_{j}
$$

so that the demand for provider $i$ is:

$$
x_{i}^{D}(t)=\frac{1}{2}+\frac{q_{i}(t)-q_{j}(t)}{2 \tau}
$$

The demand is consistent with the assumptions of uniform consumer distribution (with mass 1), exogenous locations of providers and full market 
coverage.

We propose here to introduce the assumption that the linear space $S$ is administratively divided into two regions, called $R_{1}$ and $R_{2}$ : consumers located between 0 and $1 / 2$ belong to region $R_{1}$, and consumers located between $1 / 2$ and 1 belong to region $R_{2}$. The provider located at 0 and 1 , will be indexed by 1 and 2, respectively, as they are under the administrative control of regulator of $R_{1}$ and $R_{2}$, respectively. Thus, each region has one regulator and one provider within its administrative space.

We can split the demand for each provider into two components, corresponding to "domestic" and "extra-regional" demand; formally: $x_{i}^{D}=$ $x_{i}^{i}+x_{i}^{j}$, where $x_{i}^{i}$ is the domestic demand (demand from residents in $R_{i}$ met by the provider located in the same $R_{i}$ ) and $x_{i}^{j}$ is the demand for provider $i$ coming from residents in Region $j$. Thus, we have:

$$
\begin{cases}x_{i}^{i}=\frac{1}{2}-\frac{q_{j}-q_{i}}{2 \tau}, x_{i}^{j}=0 & \text { if } q_{i} \leq q_{j} \\ x_{i}^{i}=\frac{1}{2}, x_{i}^{j}=\frac{q_{i}-q_{j}}{2 \tau} & \text { if } q_{i}>q_{j}\end{cases}
$$

that is,

$$
x_{i}^{i}=\min \left(\frac{1}{2}, \frac{1}{2}-\frac{q_{j}-q_{i}}{2 \tau}\right), \quad x_{i}^{j}=\max \left(0, \frac{q_{i}-q_{j}}{2 \tau}\right) .
$$

Following available models, we assume that the cost function of each provider is linear in the quantity, and quadratic in the quality levels $(q)$ and investment to improve quality levels $(I)$; cost may also include a fixed cost:

$$
C_{i}=c_{i} x_{i}+\frac{\beta}{2} q_{i}^{2}+\frac{\gamma}{2} I_{i}^{2}+F_{i}
$$


where $c_{i}, \beta, \gamma$ and $F_{i}$ are positive parameters. Notice that we assume that constant marginal costs $c_{i}$ and fixed cost $F_{i}$ may differ across regions; this corresponds to the fact that institutional (organizational) aspects matter on the cost structure (and it is well known that differences in efficiency between hospitals in different regions exist). Parameters $\beta$ and $\gamma$ are assumed to be equal across regions, to easy the analytics. In what follows, $\gamma$ is normalized to 1 without loss of generality. Admittedly, the functional form of the cost function is very easy and convenient for analytical reasons (separable in quantity and quality; linear in quantity and quadratic in quality and investment); however, it is commonly used in theoretical literature, and supposed to be an acceptable representation of providers' cost structure, at least under specific circumstances. Indeed, the form of cost function (i.e., linear or quadratic in quantity) corresponds to specific characteristics of service organization and institutional arrangements. In more regulated systems, like UK, Spain or Italy, there are typically excess demand and capacity constraints, which are captured by convex cost. This is not the case in systems with excess capacity (e.g., the US), where the linearity assumption seems to be more realistic. A justification for the quadratic form in quality and investment is that a higher quality level makes the required maintenance operations more demanding, and requires more skilled (i.e., more expensive) personnel. Again, complementarity between machinery and personnel, as well as links between quality levels and required investments, would suggest that separability in cost function is a disputable assumption as far as its 
realism is concerned; however, these complementarity links are far to be a strong evidence from empirical analyses (see Brekke et al. [6] and Cellini et al. [12] and the references therein for a comprehensive discussion on the meaning of linearity vs. convexity and separability of cost function in the case of healthcare service providers).

Each hospital receives a price $p_{i}$ (fixed by the domestic regional regulator) for each unit of produced service consumed by domestic patients, while the price for extra-regional treatment, $p$, is exogenously set by a central authority. This set of assumptions is consistent with what happens in the health system of several countries, like Italy or Spain, where regional regulators set the price for domestic treatment, while a system of centrally fixed prices hold for extra-regional treatments; moreover, hospitals may receive from the domestic regulator a possible lump-sum transfer to break-even, if the operative profit is negative.

Following the mentioned differential game literature, we assume that that demand is decided by each consumer at each instant of time $t \in[0,+\infty)$. The services' quality levels move over time, thanks to investment $I$ aimed at improving quality. At the beginning, quality levels are $q_{1}(0)=q_{0,1}>$ $q_{2}(0)=q_{0,2}>0$, i.e., the quality level of the provider located in $R_{1}$ is higher than the quality level of provider of $R_{2}$. Then, the dynamics of quality is ruled by the following equation:

$$
\dot{q}_{i}=I_{i}-\delta q_{i}
$$

where $\delta>0$ is a depreciation rate. Note that no externalities are at work 
across regions, and the service quality level of each provider only depends on his investment efforts (apart form the initial conditions).

Models in mentioned available literature differ as far as the objective functions concern. In some models, profit-oriented (or partially altruistic or motivated) providers aim at their own maximum result, while regulator(s) care(s) about social welfare; in other models, there is no distinction between provider and regulator, as far as the objective function concerns. In the main version of the present model (Section 3), we adopt the latter (and simpler) assumption that each regulator, aiming at maximizing the social welfare of his own region, sets the quality level; a modification, where providers aiming at maximum profit set the quality levels, is developed in Section 4. Different assumptions can be considered as well in different formulations of the model.

\section{$3 \quad$ Regional regulators as quality setters}

Since we are mainly interested in studying the dynamics of regional differences in quality of offered services and the dynamics of regional mobility of consumers, we start by keeping the model as simple as possible: we assume that the regulators set the quality (in fact, they set the investments able to move the quality); ${ }^{1}$ moreover, each regional regulator takes into account the surplus of the citizens of its own region, and the profit of the hospital located within the region, along with the (public) expenditure borne by

\footnotetext{
${ }^{1}$ See Cellini and Lamantia [11] as a model, sharing some characteristics with the present one, in which regulators set miminum quality standard instead of quality levels.
} 
himself. Formally, the instantaneous objective function of Region $i$ at time $t$ is:

$$
\Pi_{i}(t)=\sigma_{i}(t)+\pi_{i}(t)-G_{i}(t)
$$

where $\sigma_{i}$ is the surplus of residents in Region $i ; \pi_{i}$ is the profit of the hospital located in region $i$ and $G_{i}$ is the public expenditure for the service, that is, the payment from the regulator to the domestic (and possibly to the extra-regional) hospital; $G_{i}$ may also include a lump-sum transfer to the provider of the same region $i$, to reach the break-even point, in the case of a negative operative profit. Since revenues for the hospital coming from domestic treatment $\left(p_{i} x_{i}^{i}\right)$ are paid by the same regulator, this sum will not appear in the objective function. In this set of assumptions we follow Siciliani et al. [18]. Differently, sums paid by a region to the other region enter the objective function of both regulators (of course, with opposite sign). Still following Siciliani et al. [18], an opportunity $\operatorname{cost} \lambda>0$ is associated to the public expenditure different from the transfer covering the payment for domestic treatment $p_{i} x_{i}^{i}$. Thus,

$$
\begin{gathered}
\sigma_{1}(t)=\int_{0}^{\frac{1}{2}}\left(v+q_{1}-\tau x\right) d x \\
\sigma_{2}(t)=\int_{\frac{1}{2}}^{\frac{1}{2}+\frac{q_{1}-q_{2}}{2 \tau}}\left(v+q_{1}-\tau x\right) d x+\int_{\frac{1}{2}+\frac{q_{1}-q_{2}}{2 \tau}}^{1}\left[v+q_{2}-\tau(1-x)\right] d x \\
\pi_{1}(t)-G_{1}(t)=-(1+\lambda)\left[c_{1}\left(\frac{1}{2}+\frac{q_{1}-q_{2}}{2 \tau}\right)+\frac{\beta}{2} q_{1}^{2}+\frac{1}{2} I_{1}^{2}\right]+p\left(\frac{q_{1}-q_{2}}{2 \tau}\right) \\
\pi_{2}(t)-G_{2}(t)=-(1+\lambda)\left[c_{2}\left(\frac{1}{2}-\frac{q_{1}-q_{2}}{2 \tau}\right)+\frac{\beta}{2} q_{2}^{2}+\frac{1}{2} I_{2}^{2}\right]-(1+\lambda) p\left(\frac{q_{1}-q_{2}}{2 \tau}\right)
\end{gathered}
$$


All the above equations hold under the assumption that $q_{1}>q_{2}$, for any $t$. Hence, the dynamic problem of Region $i$ is:

$$
\left\{\begin{array}{l}
\max _{I_{i} \geq 0} \int_{0}^{\infty} e^{-\rho t} \Pi_{i}(t) d t \\
\dot{q}_{i}=I_{i}-\delta q_{i}, q_{i}(0)=q_{0, i} \\
\dot{q}_{j}=I_{j}-\delta q_{j}, q_{j}(0)=q_{0, j}
\end{array}\right.
$$

Notice that there is no difference, in this framework, between regulator and provider: the regulator sets the provider's investment aimed to improve the quality level of the produced service. The regulator also sets the unit price for the service delivered to domestic residents. However, the entailed public expenditure, $p_{i} x_{i}^{i}$, corresponds to revenue for the provider, so that its amount is immaterial to the objective function, and prices $p_{i}(i=1,2)$ do not enter the problems (this feature disappears from more complex version of the model, such as the formulation presented in Section 4).

Quality levels affect the demand and hence the inter-regional patients' mobility, along with the production costs. Clearly, interdependence between the two regulator's problems does exist, as long as the state variable of a player enters the problem of the other player. Hence, we are in front of a differential game.

We solve this differential game under two different assumptions concerning the information set used by the players, and correspondingly we depict the equilibrium under two different solution concepts. Firstly, we find the open-loop solution, where both players are assumed to be unable to observe the evolution of state variables over time, and they compute the optimal path 
of the choice variables at the beginning of time, and then stick to this solution forever. The open-loop solution is of type $I_{i}(t)=f\left(t ; q_{0, i}, q_{0, j}\right)$. Secondly, we will find the Markovian closed-loop feedback solution, where each player is assumed to be able to observe the dynamic evolution of state variables, and the optimal value of the choice variable depends on the current value of state variables, so that the solution is of type: $I_{i}(t)=f\left(t, q_{i}(t), q_{j}(t)\right)$. Features, pros and cons of these solution concepts are widely discussed in the differential game literature (see, e.g., Dockner et al. [13]). Feedback closed-loop solution is sometimes interpreted as 'truly dynamic' as long as players take their decisions in each instant of time, while under open-loop solution players take their decision only at the initial instant of time and then simply act according to the clock. For this reason, feedback behavior rule is interpreted as a situation in which competition is harsher as compared to the open-loop rule, as long as players can respond instant by instant to the decision of their opponents.Generally, feedback closed-loop behavior is seen as more realistic than the open-loop one. However, in the specific case of healthcare industry, the open-loop behavior is potentially relevant for explaining real world as well, beyond being a theoretical framework: for instance, as noted by Cellini et al. [12], some US states have certificate of needs $(\mathrm{CON})$ for hospitals and other healthcare service providers which require that providers elaborate investment plans and commit to them $-\mathrm{a}$ situation clearly resembling the open-loop behavior rule. 


\subsection{Open-loop Solution}

We are interested in studying the dynamics of the model in the presence of asymmetry between providers. The assumption of asymmetry can be seen as a novelty with respect to available models (like Brekke et al. [4], [5] or Siciliani et al. [18] where symmetry across providers is assumed). To fix the reference point, we introduce the following Assumptions, entailing that Region 1 is the more efficient, hence with the higher quality level, and attracting consumers from the other region.

\section{Assumptions}

$$
\begin{gathered}
\tau>\frac{1}{2 \beta(\lambda+1)} \\
p<\left(c_{2}-c_{1}\right) \frac{\lambda+1}{\lambda}
\end{gathered}
$$

Of course, situation (and conclusions) could be reversed, switching Region 1 with 2 and viceversa.

Proposition 1 Under Assumptions (10)-(11), the pair of strategies $\left(I_{1}(t), I_{2}(t)\right)$, solving the following ODE system in the state-control variables, is an open- 
loop Nash Equilibrium:

$$
\left\{\begin{array}{l}
\dot{I}_{1}=(\rho+\delta) I_{1}+\frac{1}{1+\lambda}\left[\beta(\lambda+1) q_{1}+\frac{c_{1}(\lambda+1)-p}{2 \tau}-\frac{1}{2}\right] \\
\dot{I}_{2}=(\rho+\delta) I_{2}+\frac{1}{1+\lambda}\left[\beta(\lambda+1) q_{2}+\frac{q_{1}-q_{2}}{2 \tau}+\frac{\left(c_{2}-p\right)(\lambda+1)}{2 \tau}-\frac{1}{2}\right] \\
\dot{q}_{1}=I_{1}-\delta q_{1} \\
\dot{q}_{2}=I_{2}-\delta q_{2}
\end{array}\right.
$$

with initial conditions: $q_{1}(0)=q_{0,1}>q_{2}(0)=q_{0,2}>0$.

The equilibrium point of this linear system is given by:

$I_{i}^{O L}=\delta q_{i}^{O L}, \quad i=1,2$

where $q_{i}^{O L}$ are the open-loop steady state quality levels given by

$$
\begin{gathered}
q_{1}^{O L}=\frac{p+\tau-c_{1}(\lambda+1)}{2 \tau(\lambda+1)\left(\delta^{2}+\delta \rho+\beta\right)} \\
q_{2}^{O L}=\frac{2 \tau\left(\delta^{2}+\delta \rho+\beta\right)(\lambda+1)\left[\left(p-c_{2}\right)(\lambda+1)+\tau\right]+c_{1}(\lambda+1)-p-\tau}{2 \tau\left(\delta^{2}+\delta \rho+\beta\right)(\lambda+1)\left[2 \tau\left(\delta^{2}+\delta \rho+\beta\right)(\lambda+1)-1\right]}
\end{gathered}
$$

and it constitutes a saddle point.

\section{Proof. See Appendix A.}

It is interesting to note that the steady state level of the quality produced by the provider in $R_{1}$ (with the higher quality level) does not depend on the cost parameter $c_{2}$ pertaining to the provider of the other region, while the opposite does not hold: the steady-state quality produced in $R_{2}$ is affected by the cost structure of the provider with higher quality too. Technically, this is consistent with the fact that in system (12) only $c_{1}$ and $q_{1}$ appear in the dynamic equation pertaining to $I_{1}$, while both $q_{1}$ and $q_{2}$, along with 
$c_{2}$, appear in the dynamics of $I_{2}$. From a substantial point of view, this is due to the assumption that the provider of Region 1 is (and remains) the more efficient one $^{2}$. Thus, since the provider of Region 1 is aware that he serves the whole domestic market and attracts consumers from the other region, the cost structure of Region 2 does not affect the optimal choice of the provider of Region 1. On the opposite, the quality level of provider 1 , influenced by his own cost structure, affects the amount of residents of Region 2 who decide to migrate, and hence affects the optimal investment decision of provider 2 .

Consistently with the quality differential between the two providers in steady state, the steady-state inter-regional demand flow, constituted by residents of $R_{2}$ who demand the service from the provider of $R_{1}$, turns out to be equal to

$$
\left(x_{1}^{2}\right)^{O L}=\frac{q_{1}^{O L}-q_{2}^{O L}}{2 \tau}=\frac{(1+\lambda)\left(c_{2}-c_{1}\right)-\lambda p}{2 \tau\left[2 \tau(1+\lambda)\left(\delta^{2}+\delta \rho+\beta\right)-1\right]}
$$

As far as the dynamics of such inter-regional flow is concerned, we can simply notice that, since the steady state quality levels do not depend on the initial ones, we can have a higher or a lower equilibrium quality gap between the two regions with respect to the initial value, and this obviously results in a higher or lower inter-regional patients flow.

Simple comparative statics provide the following results concerning steady

\footnotetext{
${ }^{2}$ Since the considered equilibrium is the saddle point of a linear ODE system, along the (linear) stable manifold the qualities trajectories are monotonic. Therefore $q_{0,1}>q_{0,2}$ and $q_{1}^{O L}>q_{2}^{O L}$ imply $q_{1}(t)>q_{2}(t)$ for every $t \in[0, \infty)$.
} 
state allocation.

As to the effect of $\tau$ on quality levels and inter-regional migrational flow, it is easy to check that:

$$
\begin{gathered}
\frac{\partial q_{1}^{O L}}{\partial \tau}=\frac{c_{1}(1+\lambda)-p}{2 \tau^{2}(\lambda+1)\left(\delta^{2}+\rho \delta+\beta\right)} \\
\frac{\partial q_{2}^{O L}}{\partial \tau}=\frac{c_{1}(1+\lambda)-p}{2 \tau^{2}(\lambda+1)\left(\delta^{2}+\rho \delta+\beta\right)}+\frac{2(\lambda+1)\left(p-(\lambda+1)\left(c_{1}-c_{2}+p\right)\right)\left(\delta^{2}+\rho \delta+\beta\right)}{\left(2 \tau(\lambda+1)\left(\delta^{2}+\rho \delta+\beta\right)-1\right)^{2}} \\
\frac{\partial\left(x_{1}^{2}\right)}{\partial \tau}= \\
-\frac{p-(\lambda+1)\left(c_{1}-c_{2}+p\right)}{2 \tau^{2}\left(2 \tau(\lambda+1)\left(\delta^{2}+\rho \delta+\beta\right)-1\right)}-\frac{(\lambda+1)\left(p-(\lambda+1)\left(c_{1}-c_{2}+p\right)\right)\left(\delta^{2}+\rho \delta+\beta\right)}{\tau\left(2 \tau(\lambda+1)\left(\delta^{2}+\rho \delta+\beta\right)-1\right)^{2}}
\end{gathered}
$$

It results $\frac{\partial q_{1}^{O L}}{\partial \tau}<0$ if $p>c_{1}(1+\lambda)$. The economic meaning is immediate: lower travel cost entail harsher competition (as discussed in several contributions, including Siciliani et al. [18]); harsher competition leads to higher quality levels, if unit price is sufficiently high, and providers have a financial incentive to attract extra-regional demand. On the opposite, if the regulated unit price is "too low" (and financial losses are entailed by serving the market), harsher competition leads providers to exert lower efforts to increase quality, since higher demand would entail lower revenues. Our present result perfectly mimics the outcome in Siciliani et al. [18] (subsection 3.2.2), where a brief discussion is provided on cases in which policy measures aimed at increasing competition have been based on travel cost reduction, for instance by reimbursing patients who choose to move to different regions. Clearly, 
such policy measures succeed in obtaining the expected increase of quality levels, only if they are associated to sufficiently high price for the service.

Similarly, note that $\frac{\partial q_{2}^{O L}}{\partial \tau}<0$ if $p<\frac{(\lambda+1)}{\lambda}\left(c_{2}-c_{1}\right)$, which can be interpreted in the same way as explained above for the quality of the provider of $R_{1}$. Under the same parametric condition, it holds that $\frac{\partial q_{2}^{O L}}{\partial \tau}>\frac{\partial q_{1}^{O L}}{\partial \tau}$. Moreover, condition $\tau>\frac{1}{2\left(\delta^{2}+\delta \rho+\beta\right)(\lambda+1)}$ and $p<\frac{(\lambda+1)}{\lambda}\left(c_{2}-c_{1}\right)$ are sufficient to ensure that $\left(x_{1}^{2}\right)^{O L}>0$, and $\frac{\partial\left(x_{1}^{2}\right)^{O L}}{\partial \tau}<0$. This means that harsher competition (i.e., lower $\tau$ ) leads to higher quality levels in both regions, but to a larger quality differential too: the region with lower marginal cost (and higher initial quality) increases the steady state quality by a larger amount, in front of lower consumers' travel cost, so that the amount of inter-regional migration increases. If we imagine that $\tau$ can be reduced through appropriate pro-competition policies, then such policies are beneficial for the available quality levels, but they entails larger quality differences and larger patient mobility.

As to the effect of $p$, the centrally regulated price for extra-regional treatment, we have:

$$
\begin{aligned}
\frac{\partial q_{1}^{O L}}{\partial p} & =\frac{1}{2 \tau(\lambda+1)\left(\delta^{2}+\rho \delta+\beta\right)}>0 \\
\frac{\partial q_{2}^{O L}}{\partial p} & =\frac{\lambda}{2 \tau(\lambda+1)\left(\delta^{2}+\rho \delta+\beta\right)-1}+\frac{1}{2 \tau(\lambda+1)\left(\delta^{2}+\rho \delta+\beta\right)} \\
\frac{\partial\left(x_{1}^{2}\right)^{O L}}{\partial p} & =-\frac{\lambda}{2 \tau\left(2 \tau(\lambda+1)\left(\delta^{2}+\rho \delta+\beta\right)-1\right)}
\end{aligned}
$$

An increase of the price for extra-regional treatment leads to higher quality levels in the most efficient region, that attracts extra-regional demand. 
The same effect on the quality in the less efficient region occurs only if condition $\tau>\frac{1}{2\left(\delta^{2}+\delta \rho+\beta\right)(\lambda+1)}$ is met. Under the same condition it results: $\frac{\partial\left(x_{1}^{2}\right)^{O L}}{\partial p}<0$. Verbally, under high transport cost, an increase of the price for extra-regional treatment leads the region with lower quality level to exert higher effort to increase its quality, in order to reduce the number of its citizens who decide to migrate to buy the service; on the opposite, if transportation costs are low, the less efficient region finds it convenient to reduce its quality level, inducing its citizens to buy the service from the provider of the other region.

Though rather intuitive, this result has a theoretical importance and originality, as well as a relevance as to the policy implications. It makes clear that the effect of the regulated prices upon the service quality crucially depends on the level of travel cost: if travel cost is low, an increase of regulated price level leads to lower quality in the less efficient region; the reverse occurs under high travel cost. Hence, policy interventions on travel costs (such as the reimbursement of travel cost as regulated in a few European countries for patient mobility) affect the effectiveness of price policy upon service quality.

\subsection{Feedback Solution}

Assumptions We must again assume that $\tau$ is greater than the threshold specified in (10). In addition to this, it must also hold:

$$
\alpha_{1}>k_{1}>0
$$


where the values for $\alpha_{1}$ and $k_{1}$ are given in the following Proposition, which provides a linear Feedback Nash Equilibrium of the considered differential game.

Proposition 2 A linear FNE is given by the following couple of strategies $\left(I_{1}, I_{2}\right)$ :

$$
\begin{gathered}
I_{1}=\frac{1}{1+\lambda}\left(\alpha_{1}+\alpha_{3} q_{1}\right) \\
I_{2}=\frac{1}{1+\lambda}\left(k_{1}+k_{3} q_{2}+k_{5} q_{1}\right)
\end{gathered}
$$

where, defining ${ }^{3}$ :

$$
A:=\tau(\lambda+1)\left[4 \beta+(2 \delta+\rho)^{2}\right]-2>0
$$

we have:

$$
\begin{gathered}
\alpha_{3}=\frac{\lambda+1}{2}\left[(2 \delta+\rho)-\sqrt{4 \beta+(2 \delta+\rho)^{2}}\right] \\
k_{3}=\sqrt{\frac{\lambda+1}{4 \tau}}[\sqrt{\tau(\lambda+1)}(2 \delta+\rho)-\sqrt{A}] \\
k_{5}=\sqrt{\frac{\lambda+1}{4 \tau}}[\sqrt{A}-\sqrt{A+2}]=-k_{4} \\
\alpha_{1}=\frac{N_{\alpha_{1}}}{D_{\alpha_{1}}}, \quad k_{1}=\frac{N_{k_{1}}}{D_{k_{1}}}
\end{gathered}
$$

with:

$$
\begin{gathered}
N_{\alpha_{1}}=\sqrt{\tau}(\sqrt{A}+\rho \sqrt{\tau} \sqrt{\lambda+1}) \sqrt{4 \beta+\left(2 \delta^{2}+\rho\right)^{2}}-\rho \sqrt{\tau} \sqrt{A}+ \\
+4 \beta \sqrt{\lambda+1}\left(p-c_{1}(\lambda+1)\right)-\sqrt{\lambda+1}\left(4 c_{1} \delta(\delta+\rho)(\lambda+1)-4 \delta^{2} p-\rho(4 \delta p-\rho \tau)\right)
\end{gathered}
$$

\footnotetext{
${ }^{3}$ The inequality $A>0$ follows from Assumption (10).
} 


$$
\begin{gathered}
D_{\alpha_{1}}=4 \sqrt{\tau}(\beta+\delta(\delta+\rho))(\sqrt{A}+\rho \sqrt{\tau(\lambda+1))} \\
N_{k_{1}}=\sqrt{\tau\left(4 \beta+4 \delta^{2}+4 \delta \rho+\rho^{2}\right)}\left[\rho \sqrt{A \tau(\lambda+1)}+2 \beta(\lambda+1)\left(c_{1}(\lambda+1)-p+\tau\right)+2 c_{1} \delta(\delta+\rho)(\lambda+1)^{2}+\right. \\
\left.\quad+2 \delta^{2}(\lambda+1)(\tau-p)+2 \delta \rho(\lambda+1)(\tau-p)+\rho^{2} \tau(\lambda+1)-1\right]+ \\
-\left[\sqrt { A ( \lambda + 1 ) } \left(2 \beta\left(c_{1}(\lambda+1)+\lambda\left(c_{2}-p\right)-2 p+c_{2}\right)+2 c_{1} \delta(\delta+r)(\lambda+1)-2 \delta^{2}\left(\lambda\left(p-c_{2}\right)+2 p-c_{2}\right)+\right.\right. \\
\left.\left.\left.-\rho\left(2 \delta\left(\lambda\left(p-c_{2}\right)+2 p-c_{2}\right)-\rho \tau\right)\right)-\rho \sqrt{\tau}\left(2(\lambda+1)\left((\lambda+1)\left(p-c_{2}\right)-\tau\right)\right)\left(\beta+\delta^{2}+\delta \rho\right)-\rho^{2} \tau(\lambda+1)+1\right)\right] \\
D_{k_{1}}=4 \sqrt{\tau}\left(\rho \sqrt{\tau}(\beta+\delta(\delta+\rho)) \sqrt{A(\lambda+1)}+2 \beta^{2} \tau(\lambda+1)+\beta\left(\tau(\lambda+1)(2 \delta+\rho)^{2}-1\right)+\right. \\
\left.+\delta\left(2 \delta^{3} \tau(\lambda+1)+4 \delta^{2} \rho \tau(\lambda+1)+\delta\left(3 \rho^{2} \tau(\lambda+1)-1\right)+\rho\left(\rho^{2} \tau(\lambda+1)-1\right)\right)\right)
\end{gathered}
$$

Proof. See Appendix B. Steady state qualities are thus given by:

$$
\begin{aligned}
& q_{1}^{F}=\frac{\alpha_{1}}{\delta(\lambda+1)-\alpha_{3}} \\
& q_{2}^{F}=\frac{k_{1}+k_{5} q_{1}^{F}}{\delta(\lambda+1)-k_{3}}
\end{aligned}
$$

The equilibrium point $\left(q_{1}^{F}, q_{2}^{F}\right)$ is a stable node (see Appendix B).

Remark 1 Notice that Assumption (10) ensures $k_{3}<0$, which in turn implies that the first order condition provides a maximum point for the RHS of the HJB equation of the less efficient hospital (see Appendix B). 
From (14) and (15) it follows that

$$
q_{1}^{F}>q_{2}^{F} \Longleftrightarrow q_{1}^{F}>\frac{k_{1}}{\delta(\lambda+1)-\left(k_{3}+k_{5}\right)}
$$

Therefore, recalling that $0>\alpha_{3}=k_{3}+k_{5}$, a sufficient condition for $q_{1}^{F}>q_{2}^{F}$ is: $\alpha_{1}>k_{1}$. In Appendix $B$ we prove that the considered assumption is also a sufficient condition for $q_{1}^{F}(t)>q_{2}^{F}(t), \forall t \in[0, \infty)$.

Remark 2 Notice that, since $k_{5}<0$, a negatively signed link exists between $q_{1}^{F}$ and $q_{2}^{F}$. The mathematical reason rests on the fact that $\dot{q}_{2}$ is linked to $k_{5} q_{1}$ and hence negatively to $q_{1}$.Substantially, and verbally, this means that the steady state quality of the less efficient provider is negatively related to the steady state quality of the more efficient provider.

From (17)-(20) it can be easily shown that $k_{3}<0, k_{4}>0, k_{5}<0$ and $\alpha_{3}<0$.Thus, from (14) and (15) it is immediate to see how changes in the quality levels affect the linear feed-back strategies: $I_{1}(t)$ is the lower, the higher is $q_{1}(t)$ while it is not directly affected by $q_{2}(t)$, while $I_{2}(t)$ is the lower, the higher are $q_{2}(t)$ and $q_{1}(t)$. Hence, investment of provider 2 and quality of provider 1 are intertemporal strategic substitutes according to the Jun and Vives [15] terminology: the control of provider 2 responds negatively to the state pertaining to the other player. The opposite does not holds simply because $q_{1}^{F}(t)>q_{2}^{F}(t)$ so that there is no flow from region 1 to region 2, and the provider of region 1 is not influenced by the (lower) quality in the other region. We can also briefly describe the qualitative 
behavior of quality levels equilibrium trajectories ${ }^{4}$. As for the most efficient region, the quality level is monotonic over time, obviously increasing (resp. decreasing) if $q_{0,1}<(>) q_{1}^{F}$. The equilibrium trajectory of the less efficient provider can be monotonic, but it can also increase up to a maximum point and then decrease or vice versa, depending on the initial quality levels and the steady-state ones of both the regions. However, it is interesting to notice that the differences in quality, consequently the inter-regional migrational flow over time, is a monotonic increasing (resp. decreasing) function if the initial quality difference is lower (higher) than the steady-state one.

However, in this framework with feedback information structure, the analytic expressions for the steady-state qualities are really cumbersome; hence, we confine ourselves to a few results, regarding the most efficient provider, that can be obtained analytically.

It holds:

$$
\frac{\partial q_{1}^{F}}{\partial p}=\frac{2}{\sqrt{\tau(\lambda+1)}\left(\sqrt{4 \beta+(2 \delta+\rho)^{2}}-\rho\right)(\sqrt{A}+\rho \sqrt{\tau(\lambda+1))}}>0
$$

It is interesting to note that an increase of the exogenous price of extraregional treatment has a clear and positive impact on the quality of the service provided in $R_{1}$, the region with the higher quality level and hence attracting the extra-regional demand, while the effect can be positive or negative upon the quality of the service provided in $R_{2}$, the region with the lower quality level. The intuition runs as follows. The region with the higher quality has a clear incentive to increase the amount of extra-regional

\footnotetext{
${ }^{4}$ The following results are proved in Appendix B.
} 
treatment, since the marginal gain is positive and increasing in $p$. The region with lower quality level, that has to pay for extra-regional treatments, could find it convenient to reduce the extra-regional patients' flow, or to increase it, depending on the relation between parameters connected with costs (transport cost, operative cost, opportunity cost).

As for the comparison with the open-loop steady-state quality, we have:

$q_{1}^{F}-q_{1}^{O L}=\frac{\left(p-c_{1}(\lambda+1)\right)(\sqrt{A+2}-\sqrt{A})}{2 \tau[\beta+\delta(\delta+\rho)](\lambda+1)(\sqrt{A}+\rho \sqrt{\tau(\lambda+1)})}>0 \Longleftrightarrow p>c_{1}(\lambda+1)$

The result emerges, according to which the stronger competition entailed by the feedback behavior rule (as compared to open-loop rule) leads to a higher level of steady state quality, in the region with the higher quality level, at least for sufficiently high price level of extra-regional treatment. However, this conclusion does not apply, at least in general, to the provider with the lower quality level.

\subsection{Numerical Analysis}

Resorting to numerical analysis, here we show how steady state consumer surplus and provider profits change in response to regulated price changes. All the simulation exercises are run under the following set of assumptions: $v=1, \lambda=0.2, \beta=1, \delta=0.05, \rho=0.01$. Then, we consider a benchmark case in which $c_{1}=0.8$ and $c_{2}=1.2$ (as usual, the provider of Region 1 is the more efficient one) and $\tau=0.9$, and two alternative cases, with lower transportation cost and lower cost asymmetry, respectively (See Table 1). In each of the three considered cases, there is an admissible range for $p$, which 
meets all the assumptions introduced in the theoretical model; in particular, the upper-bound is dictated by Assumption (11) while the lower-bound is determined by a joint consideration of all constraints, including $\alpha_{1}>k_{1}$. The specified feasible range for $p$ is such that the steady-state quality level is positive for both providers.

\begin{tabular}{|c||c|c|c|c|}
\hline Different Cases: & $c_{1}$ & $c_{2}$ & $\tau$ & feasible range for $p$ \\
\hline \hline Benchmark & 0.8 & 1.2 & 0.9 & $(0.694,2.4)$ \\
\hline Lower transportation cost & 0.8 & 1.2 & $\mathbf{0 . 7}$ & $(0.972,2.4)$ \\
\hline Lower cost asymmetry & $\mathbf{0 . 9}$ & $\mathbf{1 . 1}$ & 0.9 & $(0.93,1.2)$ \\
\hline
\end{tabular}

Table 1: Parameters' setup in three different cases.

Figure 1 shows steady state profits and consumer surplus in the benchmark case. Notice that consumer surplus is always increasing in $p$ : as already mentioned, this is due to the fact that the market is fully covered, and a higher price definitely leads the provider of the more efficient region to higher quality; we are here in the case where also provider of Region 2 finds it convenient to provide higher quality, which definitely increases consumers' surplus in both regions. It is more interesting to note that the consumer surplus differential (between the more and the less efficient region) increases in $p$ under the closed-loop behavior rule, while it decreases under the open-loop rule. From a policy perspective, this suggests that increas- 
ing regulated price may result in higher or lower patient surplus differential across regions, depending on how harsh is the competition among providers: in the numerical case at hand, differential enlarges under the harsher competition (associated to closed-loop behavior), while it diminishes under the milder competition (associated to open-loop behavior). As to individual steady-state profits, note that the more efficient provider has a higher profit than the less efficient one, regardless of the adopted behavior rule (openor closed-loop), while the individual profit of given provider may be larger or smaller under the open-loop w.r.t. the closed-loop behavior; individual profits for a given provider, and the consumers' surplus of the residents in a given region coincide under open- and closed-loop behavior if $p=c_{1}(\lambda+1)$ : in formal terms, for $p=c_{1}(\lambda+1)=0.96$ (dotted vertical line) it results that $\pi_{i}^{O L}=\pi_{i}^{F}, \sigma_{i}^{O L}=\sigma_{i}^{F}$ and $\Pi_{i}^{O L}=\Pi_{i}^{F}$ for $i=1,2$.

Figure 2.a,b report quality difference in steady state as a function of regulated price, confronting the benchmark case, with the case of lower transportation cost and lower cost asymmetry. From panel a, one notices that quality differential increases (decreases) in price under the closed- (open-) loop behavior; lower transportation costs do not modify the pattern of this differential, but make its size larger. Panel b shows that a smaller difference in cost efficiency of providers leads to a smaller differential in steady-state quality levels; however, the patterns of quality differential do not modify substantially, in front of (small) change in efficiency differential. It makes also clear that closed-loop behavior leads to larger steady-state quality dif- 
ferential only in the presence of high level of regulated price. In other words, stronger competition in the form of feedback behavior of providers, instead of open-loop behavior, leads to a larger quality differential between regions and hence to larger inter-regional patients' flow, in the presence of a high level of regulated price. On the contrary, larger quality differential in steady state are observed under the milder form of competition associated to openloop behavior rule, if the regulated price level is relatively low.

The richness of results should suggest that a great deal of caution is necessary when the implications of policy measures in the industry of healthcare are analysed.

\section{Extension: a model with providers as quality setters}

In the version of the model above, prices $p_{1}$ and $p_{2}$ do not enter the problem of the regulators, as they have only redistributive effects. This assumption can be easily removed, to show that the levels of price set at the regional level may have relevant impact on quality (and hence demand). An easy way to show this point is to assume, as in Brekke et al. [4], that the providers choose the investment level at each instant in time, taking price $\left(p_{1}\right.$ and $p_{2}$, in this case) as known; in such a case, objective function to maximize for provider $H_{i}$ is:

$$
J_{i}=\int_{0}^{\infty} \pi_{i}(t) e^{-\rho t} d t
$$


where instantaneous profit is:

$$
\pi_{i}=\left(p_{i}-c_{i}\right) x_{i}^{D}-\frac{\gamma}{2} I_{i}^{2}-\frac{\beta}{2} q_{i}^{2}
$$

Notice that provider $i$ receives $p_{i}$ for each case treated; however, Region $i$ pays for each consumer belonging to $R_{i}$, while the same amount $p_{i}$ is paid from region $R_{j}$ for any patient belonging to $x_{i}^{j}$. Notice also that, for the sake of easiness, we assume that the objective function of each provider is simply its own profit, without taking into account the wide body of considerations supporting the occurrence of motivated (i.e., semi-altruistic) providers (see, e.g., Siciliani et al. [18] and references therein).

As in the model presented in the previous section, control variable is investment in quality, which affects the quality level, according to equation (5), again given an initial condition $q_{0,1}>0, q_{0,2}>0$. The following propositions provide the open-loop Nash equilibrium (OLNE) and the linear closed-loop feedback Nash equilibrium (FNE) of the model into consideration.

Proposition 3 The ODE system in the state-control variables that an openloop Nash Equilibrium strategies pair $\left(I_{1}(t), I_{2}(t)\right)$ solves is given by:

$$
\left\{\begin{array}{l}
\dot{I}_{i}=(\rho+\delta) I_{i}+\beta q_{i}-\frac{p_{i}-c_{i}}{2 \tau} \\
\dot{q}_{i}=I_{i}-\delta q_{i}
\end{array}\right.
$$

with $i=1,2$. The equilibrium point of this linear system is given by:

$$
\begin{aligned}
I_{i}^{O L} & =\delta q_{i}^{O L}, \quad i=1,2 \\
q_{i}^{O L} & =\frac{p_{i}-c_{i}}{2 \tau\left(\delta^{2}+\delta \rho+\beta\right)}
\end{aligned}
$$


and it constitutes a saddle point.

Proof. The proof is omitted since it is analogous to that provided in Brekke et al. [4] for the case of constant marginal treatment costs with $\varphi=0$ and $\gamma=1$ but allowing for different coefficients $p_{i}$ and $c_{i}$.

Proposition 4 A linear FNE strategy for provider $H_{i}, i \in\{1,2\}$, is given by:

$$
I_{i}(t)=\varphi_{i}+\omega q_{i}(t)
$$

where:

$$
\begin{gathered}
\varphi_{i}=\frac{p_{i}-c_{i}}{\tau \sqrt{4 \beta+(\rho+2 \delta)^{2}}} \\
\omega=\delta+\frac{\rho}{2}-\sqrt{\beta+\left(\delta+\frac{\rho}{2}\right)^{2}}
\end{gathered}
$$

Proof. The proof is omitted since it employs the same technique shown in Appendix B and it yields a solution analogous to that obtained by Brekke et al. [4] for the considered case with different treatment marginal costs $c_{i}$ and prices $p_{i}$ across hospitals.

Note that our solution constitutes the only linear FNE in which the control chosen, in every time instant, by each hospital depends only on the current value of its own quality level (and on the price chosen by its Region).

From the FNE strategies, by using the state variable dynamic, we obtain the time-path of the quality level for $H_{i}$ :

$$
q_{i}(t)=\left[q_{0, i}-\frac{p_{i}-c_{i}}{2 \tau\left(\delta^{2}+\beta+\rho \delta\right)}\right] e^{\left(\frac{\rho}{2}-\sqrt{\beta+\left(\frac{\rho}{2}+\delta\right)^{2}}\right) t}+\frac{p_{i}-c_{i}}{2 \tau\left(\delta^{2}+\beta+\rho \delta\right)}
$$


Sufficient condition for $q_{i}(t)>0$ is $p_{i}>c_{i}$. It holds:

$$
\Delta q(t)=\left[\Delta q^{0}-\frac{\Delta p-\Delta c}{2 \tau\left(\delta^{2}+\beta+\rho \delta\right)}\right] e^{\left(\frac{\rho}{2}-\sqrt{\beta+\left(\frac{\rho}{2}+\delta\right)^{2}}\right) t}+\frac{\Delta p-\Delta c}{2 \tau\left(\delta^{2}+\beta+\rho \delta\right)}
$$

where:

$\Delta q(t):=q_{1}(t)-q_{2}(t), \quad \Delta q^{0}:=q_{0,1}-q_{0,2}, \quad \Delta p:=p_{1}-p_{2}, \quad \Delta c:=c_{1}-c_{2}$.

Since we have: $\frac{\rho}{2}-\sqrt{\beta+\left(\frac{\rho}{2}+\delta\right)^{2}}<0$, in steady-state it holds that: $\Delta q>$ $0 \Longleftrightarrow \Delta p>\Delta c$.

Let us consider, without loss of generality: $\Delta q^{0}>0$. Consequently, as in the model presented in Section 3, it is realistic to consider also $\Delta c<0$. It holds:

1. if $\Delta p>\Delta c$ then $\forall t \in[0, \infty): \Delta q(t)>0$;

2. if $\Delta p<\Delta c$ then $\Delta q(t)>0$ for all $t<\bar{t}$, where:

$$
\bar{t}=\frac{\ln \left(\frac{\Delta p-\Delta c}{(\Delta p-\Delta c)-\Delta q_{0}\left[2 \tau\left(\delta^{2}+\beta+\rho \delta\right)\right]}\right)}{\left(\frac{\rho}{2}-\sqrt{\beta+\left(\frac{\rho}{2}+\delta\right)^{2}}\right)}
$$

while for all $t>\bar{t}$ we have: $\Delta q(t)<0$. Not surprisingly, $\bar{t}$ is increasing in the initial quality difference and in the marginal disutility of traveling and it is decreasing in the term $\Delta p-\Delta c$.

Finally we notice that, as in the symmetric model of Brekke et al. [4], and differently from the model presented in the previous section, the openloop and the feedback solutions lead to the same steady-state values. 
The interest of the version of the model presented in this section rests in the fact that it shows that quality differential between regions may switch its algebraic sign at a point in time, in front of specific parameters' configuration: a "too low" regulated price level may induce the more efficient provider to select investment plans that lead to a lower quality as compared to the other region's provider. The further step left to future research, consists in considering regional prices as endogenous, and set by the regulators in order to maximize a social welfare function, different from the objective function of providers.

\section{Concluding remarks}

In this paper we have proposed a differential game model to highlight the role of local regulation in a market where prices are given and competition is based on quality. We have shown that quality differential across region can persist, under equilibrium conditions. We have studied the relations between regulated price levels, and quality levels and dynamics. Of course, quality levels affect demand and hence inter-regional mobility. Our theoretical model has a clear empirical counterpart in healthcare markets, as well as in other markets like childcare, long-term care, or even education, especially at the primary and secondary school levels, with very similar characteristics as healthcare: competition among providers bases on quality rather than price; consumers' choices are driven by location and quality of providers.

From a mathematical viewpoint, both the model of Section 3 and its 
variant in Section 4 are linear-quadratic differential games with one control variable for each player $\left(I_{i}\right)$ and two state variables $\left(q_{1}\right.$ and $\left.q_{2}\right)$, which have very simple dynamics. The setting is simple enough to let us to derive analytically the open-loop and the linear Feedback Nash Equilibria of those games. However, it is interesting to notice that they are not linear-state games (see Dockner et al. [13]), since the instantaneous pay-off functions are not linear in the state variables: this implies that the open-loop Nash equilibria are not sub-game perfect. In particular we have a saddle-point equilibrium in both the models, implying that, given the initial quality levels, we can find initial values of the co-state variables (which in turn determine the initial optimal investment choices) such that the system converges to the steady state as time approaches infinity. Conversely, in the corresponding games with feedback information pattern, we considered the stationary linear closed-loop feedback equilibrium (which is, by definition, a sub-game perfect equilibrium) which stabilizes the states for every possible initial condition. Interestingly, these two different equilibrium paths lead to the same steady-state framework in the model of Section 4, while this is not the case for the model of Section 3. In both the models there is asymmetry between the players, but the degree of asymmetry is much bigger in the first one, in which the players ex-ante "know" that the most efficient one will always have higher quality levels (so they have different objective functionals form), and this leads to the feature that -for both the obtained equilibria- the equilibrium strategy of this player -differently from 
what happens for the less efficient one- does not explicitly depend on the state variable of the other one. Conversely, the model presented in Section 4, which is a generalization of Brekke et al. [4] work, is characterized by the fact that both in the open-loop and in the linear feedback Nash equilibrium with asymptotically stable steady state, the strategy of player $i$ does not explicitly depend on the state variable of player $j$. In this simpler setting we have also investigated under which conditions an equilibrium trajectory with inversion of quality levels can occur. Specific assumptions concerning the choice variables of regulators lead to different properties of equilibria. From a simple comparison between the models of Section 3 and 4, one can see that the steady state under open-loop vs. feedback behavior rule may coincide or not, depending on whether the quality is chosen by the regulator or by the (profit-oriented) provider.

Our model can be extended along different routes. The most immediate one is to make the regulated prices endogenous, imagining that prices are set to maximize some social welfare functions in which regulated prices matter. In the present version of the model, local prices do not enter the objective functions of local regulators, and they have a mere redistributive role.

Though very simple, the present version of the model can provide some policy prescriptions. For instance, pro-competition policies are effective in fostering available quality of services only under specific conditions (as shown, by the way, by other available models with different focuses from regional distribution of quality levels and inter-regional consumers' mobility); 
more important, out present model shows that pro-competition policies may lead to increasing or decreasing quality differential across regions, depending on the parameter configuration, that is, the initial framework configuration, and also on the behavior rule followed by providers (namely, open-loop vs closed-loop behavior rules). Thus, our model suggests that the outcome of pro-competitive policy measures (which have been very popular over the past decades in several western countries, as applied to markets characterized by quality competition) are far from having obvious and similar results across different countries and institutional contexts.

\section{Acknowledgements}

We thank Rosella Levaggi, Luca Lambertini and Luigi Siciliani for helpful comments on a previous draft of this article. We also thank two anonymous referees for their suggestions. The responsibility remains on the Authors only.

\section{Appendix}

\section{A Proof of Proposition 1}

In order to find an open-loop Nash equilibrium for the considered differential game, we apply the Pontryagin maximum principle. The current Hamilto- 
nian function of player $i, i \in\{1,2\}$, is given by $^{5}$ :

$$
H_{i}=\Pi_{i}+\mu_{i}\left(I_{i}-\delta q_{i}\right)+\phi_{i}\left(I_{j}-\delta q_{j}\right)
$$

where $\mu_{i}$ and $\phi_{i}$ are the current co-state variables and the instantaneous profit functions $\Pi_{i}$ are obtained by considering equations (6),(7),(8),(9). The adjoint equations are given by:

$$
\begin{gathered}
\dot{\mu}_{1}=\rho \mu_{1}-\frac{\partial H_{1}}{\partial q_{1}} \Longrightarrow \dot{\mu}_{1}=(\rho+\delta) \mu_{1}+\beta(\lambda+1) q_{1}+\frac{c_{1}(\lambda+1)-p}{2 \tau}-\frac{1}{2} \\
\dot{\phi}_{1}=\rho \phi_{1}-\frac{\partial H_{1}}{\partial q_{2}} \Longrightarrow \dot{\phi}_{1}=(\rho+\delta) \phi_{1}-\frac{c_{1}(1+\lambda)-p}{2 \tau} \\
\dot{\mu}_{2}=\rho \mu_{2}-\frac{\partial H_{2}}{\partial q_{2}} \Longrightarrow \dot{\mu}_{2}=(\rho+\delta) \mu_{2}+\beta(\lambda+1) q_{2}+\frac{q_{1}-q_{2}}{2 \tau}+\frac{\left(c_{2}-p\right)(\lambda+1)}{2 \tau}-\frac{1}{2} \\
\dot{\phi}_{2}=\rho \phi_{2}-\frac{\partial H_{2}}{\partial q_{1}} \Longrightarrow \dot{\phi}_{2}=(\rho+\delta) \phi_{2}-\frac{\left(c_{2}-p\right)(1+\lambda)+q_{2}-q_{1}}{2 \tau}
\end{gathered}
$$

Transversality conditions are: $\lim _{t \rightarrow \infty} e^{-\rho t} \mu_{i} q_{i}=0, \lim _{t \rightarrow \infty} e^{-\rho t} \phi_{i} q_{j}=0$. First order conditions for a maximum point of the Hamiltonian functions give:

$$
\frac{\partial H_{i}}{\partial I_{i}}=0 \Longleftrightarrow \mu_{i}=(1+\lambda) I_{i}
$$

Please note that the second order conditions are satisfied if the Hamiltonian of each player is concave in its control and state variables. As well known, to this end, a necessary and sufficient condition is that their Hessian matrices $\nabla_{H_{i}}^{2}$ are negative semidefinite. Since these Hessian matrices are given by:

$$
\nabla_{H_{1}}^{2}=\left(\begin{array}{cc}
-(1+\lambda) & 0 \\
0 & -\beta(1+\lambda)
\end{array}\right)
$$

\footnotetext{
${ }^{5}$ For notational simplicity, we omit time dependencies.
} 


$$
\nabla_{H_{2}}^{2}=\left(\begin{array}{cc}
-(1+\lambda) & 0 \\
0 & \frac{1}{2 \tau}-\beta(1+\lambda)
\end{array}\right)
$$

it is trivial to see that this condition is verified under Assumption (10).

Differentiating equation (29) w.r.t. time yields:

$$
\dot{\mu}_{i}=(1+\lambda) \dot{I}_{i}
$$

which, substituted into (25) and (27), together with the quality stock dynamic equations, lead to the linear ODE system given in Proposition 1.

In this system, by imposing $\dot{I}_{i}=\dot{q}_{i}=0$, we get the equilibrium point. In order to determine its nature, we compute the eigenvalues of the coefficient matrix of the system (12):

$$
\begin{gathered}
\lambda_{1}=\frac{\rho}{2}+\sqrt{\delta^{2}+\delta \rho+\frac{\rho^{2}}{4}+\beta} \\
\lambda_{2}=\frac{\rho}{2}-\sqrt{\delta^{2}+\delta \rho+\frac{\rho^{2}}{4}+\beta-\frac{1}{2 \tau(1+\lambda)}} \\
\lambda_{3}=\frac{\rho}{2}+\sqrt{\delta^{2}+\delta \rho+\frac{\rho^{2}}{4}+\beta-\frac{1}{2 \tau(1+\lambda)}} \\
\lambda_{4}=\frac{\rho}{2}-\sqrt{\delta^{2}+\delta \rho+\frac{\rho^{2}}{4}+\beta}
\end{gathered}
$$

furthermore, under Assumption (10), it follows that all these eigenvalues are real and:

$$
\lambda_{4}<\lambda_{2}<0<\lambda_{3}<\lambda_{1}
$$

therefore we get eigenvalues with positive values and others with negative values, hence the equilibrium point constitutes a saddle point. 


\section{B Proof of Proposition 2 and Related Results}

In order $I_{i}\left(q_{i}, q_{j}\right)$, with $i, j \in\{1,2\}, i \neq j$, to be a FNE strategy for player $i$, we look for a value function $V\left(q_{i}, q_{j}\right)$, continuously differentiable, which satisfies the following Hamilton-Jacobi-Bellman (HJB) equation:

$$
\rho V_{i}=\max _{I_{i} \geq 0}\left\{\Pi_{i}+\frac{\partial V_{i}}{\partial q_{i}}\left(I_{i}-\delta q_{i}\right)+\frac{\partial V_{i}}{\partial q_{j}}\left(I_{j}-\delta q_{j}\right)\right\}
$$

The FOC for the maximum point of the RHS of this HJB equation leads to:

$$
I_{i}=\frac{1}{1+\lambda} \frac{\partial V_{i}}{\partial q_{i}}
$$

Such condition is also sufficient for a maximum point since the expression to be maximized in the HJB equation is strictly concave in the control variable.

In order to find a pair of linear stationary feedback strategies that constitute a FNE of the differential game, we look for two value functions quadratic w.r.t. $q_{1}$ and $q_{2}$ :

$$
\begin{gathered}
V_{1}\left(q_{1}, q_{2}\right)=\alpha_{0}+\alpha_{1} q_{1}+\alpha_{2} q_{2}+\frac{\alpha_{3}}{2} q_{1}^{2}+\frac{\alpha_{4}}{2} q_{2}^{2}+\alpha_{5} q_{1} q_{2} \\
V_{2}\left(q_{1}, q_{2}\right)=k_{0}+k_{1} q_{2}+k_{2} q_{1}+\frac{k_{3}}{2} q_{2}^{2}+\frac{k_{4}}{2} q_{1}^{2}+k_{5} q_{1} q_{2}
\end{gathered}
$$

where $\alpha_{0}, \ldots, \alpha_{5}, k_{0}, \ldots, k_{5}$ are unknown coefficients to be determined.

By substituting in the FOC (31) we obtain:

$$
\begin{aligned}
& I_{1}=\frac{1}{1+\lambda}\left(\alpha_{1}+\alpha_{3} q_{1}+\alpha_{5} q_{2}\right) \\
& I_{2}=\frac{1}{1+\lambda}\left(k_{1}+k_{3} q_{2}+k_{5} q_{1}\right)
\end{aligned}
$$


By substituting in the HJB equation of $R_{1}$ we obtain:

$$
\begin{gathered}
\tau\left[8 \rho(\lambda+1) \alpha_{0}-4 \alpha_{1}^{2}+4 c_{1}(\lambda+1)^{2}-8 \alpha_{2} k_{1}+(\lambda+1)(\tau-4 v)\right]+ \\
+\left[2 \tau \alpha_{1}\left[\delta(\lambda+1)-\alpha_{3}+\rho(\lambda+1)\right]+c_{1}(\lambda+1)^{2}-2 \tau \alpha_{2} k_{5}-(\lambda+1)(p+\tau)-2 \tau \alpha_{5} k_{1}\right] q_{1}+ \\
+\left[-2 \tau \alpha_{1} \alpha_{5}-c_{1}(\lambda+1)^{2}+2 \tau \delta \alpha_{2}(\lambda+1)+2 \tau \alpha_{2}\left[\rho(\lambda+1)-k_{3}\right]+p(\lambda+1)-2 \tau k_{1}\right] q_{2}+ \\
+\left[\beta(\lambda+1)^{2}+2 \delta(\lambda+1) \alpha_{3}-\alpha_{3}^{2}+\rho(\lambda+1) \alpha_{3}-2 \alpha_{5} k_{5}\right] q_{1}^{2}+ \\
+\left[2 \delta(\lambda+1) \alpha_{4}+\rho(\lambda+1) \alpha_{4}-\alpha_{5}^{2}-2 \alpha_{4} k_{3}\right] q_{2}^{2}+ \\
+\left[2 \delta(\lambda+1) \alpha_{5}-\alpha_{3} \alpha_{5}+\rho(\lambda+1) \alpha_{5}-\alpha_{5} k_{3}-\alpha_{4} k_{5}\right] q_{1} q_{2}=0
\end{gathered}
$$

Similarly, by substituting in the HJB equation of $R_{2}$ we obtain:

$$
\begin{gathered}
\tau\left[8 \alpha_{1} k_{2}-(\lambda+1)\left(8 \rho k_{0}+4 \lambda c_{2}+\tau-4\left(v-c_{2}\right)\right)+4 k_{1}^{2}\right]+ \\
+\left[2 \tau \alpha_{1} k_{4}-2 \delta \tau(\lambda+1) k_{2}+2 \tau \alpha_{3} k_{2}-(\lambda+1)\left[\lambda\left(p-c_{2}\right)+2 \rho \tau k_{2}+p-c_{2}\right]+2 \tau \alpha_{5} k_{1}\right] q_{1}+ \\
\left.+\left[-2 \tau \alpha_{1} k_{5}+2 \delta \tau(\lambda+1) k_{1}-(\lambda+1)\left[\lambda\left(p-c_{2}\right)-2 \rho \tau k_{1}+p+\tau-c_{2}\right)-2 \tau\left(\alpha_{5} k_{2}+k_{1} k_{3}\right)\right]\right] q_{2}+ \\
+\left[4 \delta \tau(\lambda+1) k_{4}-4 \tau \alpha_{3} k_{4}+(\lambda+1)\left(2 \rho \tau k_{4}-1\right)-2 \tau k_{5}^{2}\right] q_{1}^{2}+ \\
+\left[2 \beta \tau(\lambda+1)^{2}+4 \delta \tau(\lambda+1) k_{3}+(\lambda+1)\left(2 \rho \tau k_{3}-1\right)-2 \tau\left(2 \alpha_{5} k_{5}+k_{3}^{2}\right)\right] q_{2}^{2}+ \\
+\left[4 \delta \tau(\lambda+1) k_{5}-2 \tau \alpha_{3} k_{5}+\left[\lambda\left(2 \rho \tau k_{5}+1\right)+2 \rho \tau k_{5}+1\right]-2 \tau \alpha_{5} k_{4}-2 \tau k_{5} k_{3}\right] q_{1} q_{2}=0
\end{gathered}
$$

For the equality to hold, all the terms in brackets in the above equations have to be equal to zero.

We concentrate on the last three equations for both the players, which do not depend on $\alpha_{0}, \alpha_{1}, \alpha_{2}, k_{0}, k_{1}, k_{2}$, obtaining a non-linear system of 6 equations in 6 unknowns. 
A solution of this system gives:

$$
\begin{gathered}
\alpha_{4}=\alpha_{5}=0 \\
k_{4}=\sqrt{\frac{\lambda+1}{4 \tau}}[\sqrt{A+2}-\sqrt{A}]
\end{gathered}
$$

with $A$ defined by (16), and the values of $\alpha_{3}, k_{3}, k_{5}$ in equations (17), (18), (19), respectively.

From this solution we obtain the values for $\alpha_{1}$ and $k_{1}$ given in Proposition $2^{6}$. Finally, by substituting these obtained values into equations (34) and (35), we get the FNE given in Proposition 2.

In order for the obtained solution to provide a maximum point for our problem which is also globally asymptotically stable we must impose (see Brekke et al. [4]):

$$
\begin{gathered}
\alpha_{3}<0 \\
\alpha_{3}+\alpha_{5}<0 \\
k_{3}<0 \Longleftrightarrow 4 \beta \tau(\lambda+1)-2>0
\end{gathered}
$$

which results in Assumption (10).

From the FNE strategies and the quality levels dynamic equations we obtain that the FNE qualities time paths are the solutions of the following linear ODE system:

$$
\left\{\begin{array}{l}
\dot{q}_{1}=\frac{1}{1+\lambda}\left[\alpha_{1}+\left(\alpha_{3}-\delta(\lambda+1)\right) q_{1}\right] \\
\dot{q}_{2}=\frac{1}{1+\lambda}\left[k_{1}+k_{5} q_{1}+\left(k_{3}-\delta(\lambda+1)\right) q_{2}\right]
\end{array}\right.
$$

\footnotetext{
${ }^{6}$ Obviously also the values for $\alpha_{0}, \alpha_{2}, k_{0}, k_{2}$ can be uniquely determined, but we can omit them since they do not enter the expression of the FNE strategies.
} 
The eigenvalues $\lambda_{1,2}$ of its coefficient matrix $B$ are real since it holds:

$(\operatorname{tr} B)^{2}-4 \operatorname{det} B=\frac{\left(\alpha_{3}+k_{3}-2 \delta(\lambda+1)\right)^{2}}{(1+\lambda)^{2}}-4 \frac{\left(\alpha_{3}-\delta(\lambda+1)\right)\left(k_{3}-\delta(\lambda+1)\right)}{(1+\lambda)^{2}}=\frac{\left(\alpha_{3}-k_{3}\right)^{2}}{(1+\lambda)^{2}}>0$

Since the equilibrium point is globally asymptotically stable, the conclusion

$\lambda_{1,2} \in \mathbb{R}$ implies that these eigenvalues are negative, therefore the equilibrium point of the system is a stable node.

The solution of the considered ODE system is given by:

$$
\left\{\begin{array}{l}
q_{1}(t)=\frac{e^{\left(\frac{k_{3}}{\lambda+1}-\delta\right) t}\left[\alpha_{1}+q_{0,1}\left(\alpha_{3}-\delta(\lambda+1)\right)\right]-\alpha_{1}}{\alpha_{3}-\delta(\lambda+1)} \\
q_{2}(t)=e^{\left(\frac{\alpha_{3}}{\lambda+1}-\delta\right) t} \frac{\alpha_{1}+q_{0,2}\left(\alpha_{3}-\delta(\lambda+1)\right)}{\alpha_{3}-\delta(\lambda+1)}+ \\
+\frac{\alpha_{1} k_{5}-k_{1}\left[\alpha_{3}-\delta(\lambda+1)\right]-e^{\left(\frac{k_{3}}{\lambda+1}-\delta\right) t}\left[\alpha_{1}-k_{1}+\left(q_{0,2}-q_{0,1}\right)\left(\delta(\lambda+1)-k_{3}\right)\right]\left[\alpha_{3}-\delta(\lambda+1)\right]}{\left[\alpha_{3}-\delta(\lambda+1)\right]\left[k_{3}-\delta(\lambda+1)\right]}
\end{array}\right.
$$

It holds:

$$
q_{1}(t)-q_{2}(t)=\frac{\left(\alpha_{1}-k_{1}\right)\left(1-e^{-\left(\delta-\frac{k_{3}}{\lambda+1}\right) t}\right)}{\delta(\lambda+1)-k_{3}}+e^{-\left(\delta-\frac{k_{3}}{\lambda+1}\right) t}\left(q_{0,1}-q_{0,2}\right)
$$

since $k_{3}<0$ and we assumed $q_{0,1}>q_{0,2}$, it follows that $\alpha_{1}>k_{1}$ constitutes a sufficient condition for $q_{1}(t)>q_{2}(t) \forall t \in[0, \infty)$.

Furthermore, by differentiating (36) w.r.t. $t$, we get:

$$
\frac{\partial\left[q_{1}(t)-q_{2}(t)\right]}{\partial t}=\frac{e^{-\left(\delta-\frac{k_{3}}{\lambda+1}\right) t}}{\lambda+1}\left[\alpha_{1}-k_{1}-\left(q_{0,1}-q_{0,2}\right)\left(\delta(\lambda+1)-k_{3}\right)\right]
$$

Therefore:

$\forall t \in[0, \infty): \frac{\partial\left[q_{1}(t)-q_{2}(t)\right]}{\partial t}>0 \Longleftrightarrow q_{0,1}-q_{0,2}<\frac{\alpha_{1}-k_{1}}{\delta(\lambda+1)-k_{3}}=q_{1}^{F}-q_{2}^{F}$

It is trivial to see that:

$$
\frac{\partial q_{1}(t)}{\partial t}=\frac{e^{\left(\frac{\alpha_{3}}{\lambda+1}-\delta\right) t}\left[\alpha_{1}+q_{0,1}\left[\alpha_{3}-\delta(\lambda+1)\right]\right.}{\lambda+1}>(<) 0 \forall t \Longleftrightarrow q_{0,1}<(>) q_{1}^{F}
$$


As for $q_{2}(t)$, it results:

$\frac{\partial q_{2}(t)}{\partial t}=\frac{e^{\left(\frac{\alpha_{3}}{\lambda+1}-\delta\right) t}\left[\alpha_{1}+q_{0,1}\left(\alpha_{3}-\delta(\lambda+1)\right)\right]-e^{\left(\frac{k_{3}}{\lambda+1}-\delta\right) t}\left[\alpha_{1}-k_{1}+\left(q_{0,1}-q_{0,1}\right)\left[k_{3}-\delta(\lambda+1)\right]\right]}{\lambda+1}$

therefore:

$\frac{\partial q_{2}(t)}{\partial t}>0 \Longleftrightarrow e^{\frac{k_{5}}{\lambda+1} t}\left[\alpha_{1}+q_{0}^{1}\left[\alpha_{3}-\delta(\lambda+1)\right]\right]>\alpha_{1}-k_{1}+\left(q_{0,1}-q_{0,2}\right)\left[k_{3}-\delta(\lambda+1)\right]$

where the LHS is positive if and only if $q_{0,1}<q_{1}^{F}$, the RHS is positive if and only if the initial quality difference is lower than the steady-state one, i.e. if $q_{0,1}<q_{0,2}+q_{1}^{F}-q_{2}^{F}$, and we have: $q_{1}^{F}<q_{0,2}+q_{1}^{F}-q_{2}^{F} \Longleftrightarrow q_{0,2}>q_{2}^{F}$. Hence, several possibilities can arise, and the trajectory can either be monotone, or it can have a maximum/minimum point.

\section{References}

[1] Aiura H (2016), The effect of cross-border healthcare on quality, public health insurance, and income redistribution, Institute for Sammfunsokonomi Working Paper 4/2016 (SSRN wp 2766847).

[2] Balia S, Brau R, Marroccu E (2014), What drives patient mobility across Italian regions? Evidence from hospital discharge data, Development in Health Economics and Public Policy 12: 133-54.

[3] Bisceglia M, Cellini R, Grilli L (2017), Regional regulators in healthcare service under quality competition: A game theoretical model, MPRA w.p. 80507. 
[4] Brekke K, Cellini R, Siciliani L, Straume O R (2010), Competition and quality in health care markets: A differential game approach, Journal of Health Economics, 29, 508-523.

[5] Brekke K, Cellini R, Siciliani L, Straume O R (2012), Competition in Regulated Markets with Sluggish Beliefs about Quality, Journal of Economics \& Management Strategy, 21: 131-178.

[6] Brekke K, Cellini R, Siciliani L, Straume O R (2018), Differential games in health care markets: Models of quality competition with fixed prices, in Apaloo J, Viscolani B (Eds), Advances in dynamic and mean-filed games - ISDG Annals, Basel (CH): Birkhauser, forthcoming.

[7] Brekke K, Levaggi R, Siciliani L, Straume O R (2014), Patient mobility, healthcare quality and welfare, Journal of Economic Behavior \& Organization, 105: 140-57.

[8] Brekke K, Levaggi R, Siciliani L, Straume O R (2016), Patient mobility and health care quality when regions and patients differ in income, Journal of Health Economics, 50: 372-87.

[9] Brekke K, Siciliani L, Straume O R (2011), Hospital competition and quality with regulated prices, The Scandinavian Journal of Economics, 113: 444-469. 
[10] Calem P S, Rizzo J A (1995), Competition and specialization in the hospital industry: an application of Hotelling's location model. Southern Economic Journal, 61: 1182-98.

[11] Cellini, R, Lamantia F (2015), Quality competition in markets with regulated prices and minimum quality standards. Journal of Evolutionary Economics, 25: 345-70.

[12] Cellini R, Siciliani L, Straume O R (2015), A dynamic model of quality competition with endogenous prices, NIPE, Universidade do Minho.

[13] Dockner E, Jorgensen S, Van Long N, Sorger G (2000), Differential Games in Economics and Management Sciences, Cambridge University Press.

[14] Hotelling, H (1929), Stabiity in competition. The Economic Journal, 153: $41-57$

[15] Jun, B, Vives X (2004), Strategic Incentives in Dynamic Duopoly. Journal of Economic Theory: 116, 249-281.

[16] Levaggi R, Moretto M, Pertile P (2012), Static and dynamic efficiency of irreversible health care investments under alternative payment rules, Journal of Health Economics 31: 169-79.

[17] Levaggi R, Moretto M, Pertile P (2014), Two-part payments for the reimbursement of investments in health technologies, Health Policy 115: $230-36$. 
[18] Siciliani L, Straume O D, Cellini R (2013), Quality competition with motivated providers and sluggish demand, Journal of Economic Dynamics \& Control, 37: 2041-2061. 


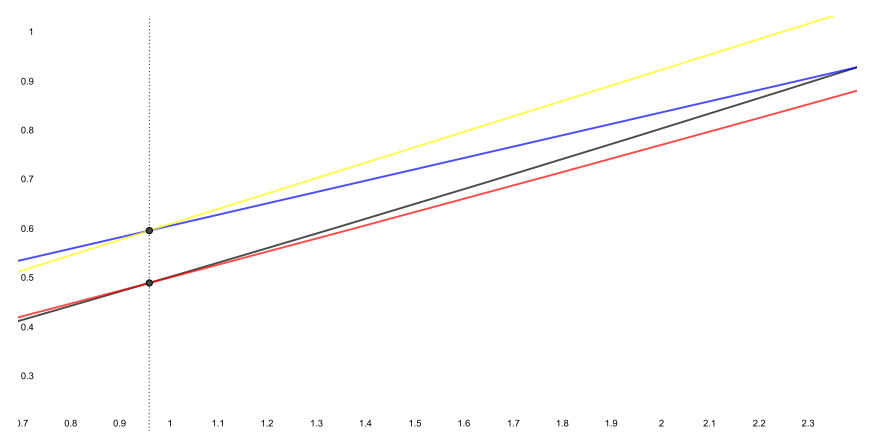

(a) Consumer Surplus: Blue $=\sigma_{1}^{O L} ;$ Black $=\sigma_{2}^{O L}$; Yellow $=\sigma_{1}^{F} ; \operatorname{Red}=\sigma_{2}^{F}$

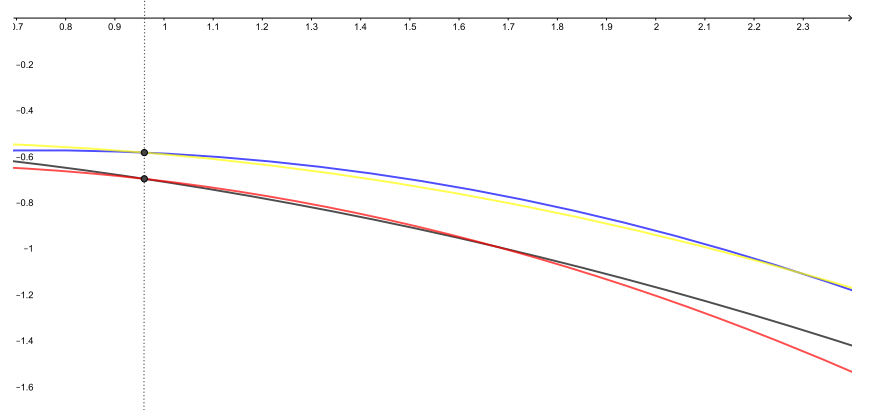

(b) Hospital Profits: Blue $=\pi_{1}^{O L} ;$ Black $=\pi_{2}^{O L} ;$ Yellow $=\pi_{1}^{F} ; \operatorname{Red}=\pi_{2}^{F}$

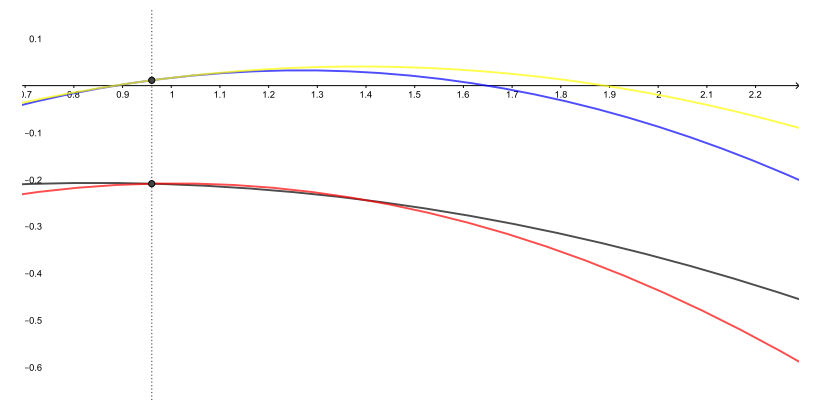

(c) Regional Social Welfare: Blue $=\Pi_{1}^{O L}$; Black $=\Pi_{2}^{O L}$; Yellow $=\Pi_{1}^{F}$;

$\operatorname{Red}=\Pi_{2}^{F}$

Figure 1: Consumer Surplus, Hospital Profits and Regional Social Welfare in steady-state for the benchmark example. 


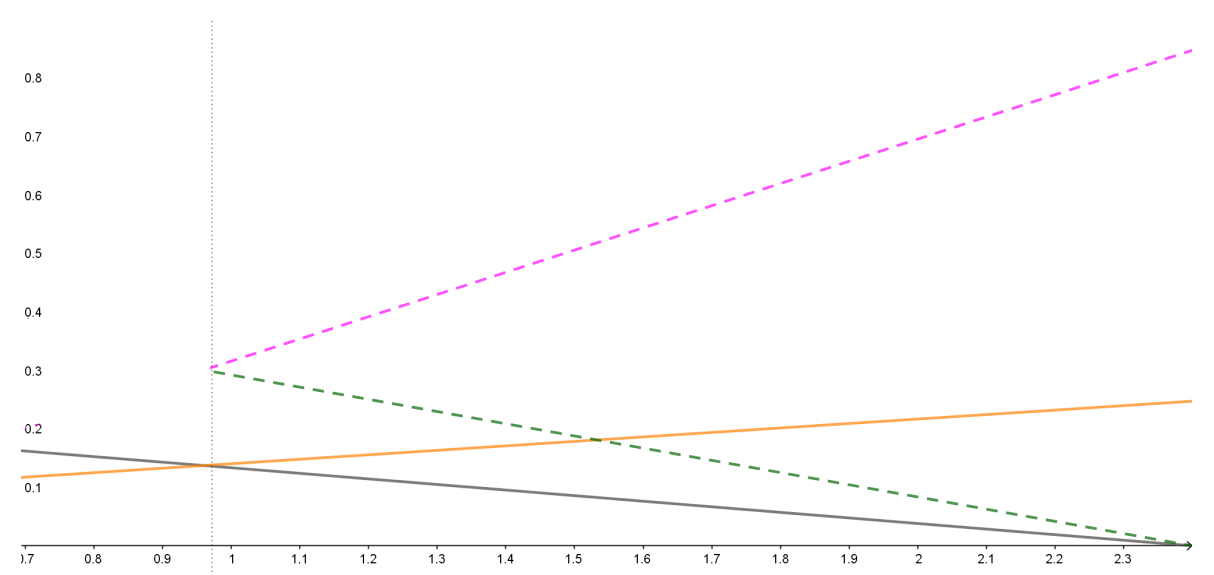

(a) Benchmark vs Lower Transportation Cost. Benchmark case $(\tau=0.9)$ : Grey $=\left(x_{1}^{2}\right)^{O L}$ and Orange $=\left(x_{1}^{2}\right)^{F}$. Lower Transportation Cost $(\tau=0.7)$ : Green $=\left(x_{1}^{2}\right)^{O L}$ and Magenta $=\left(x_{1}^{2}\right)^{F}$
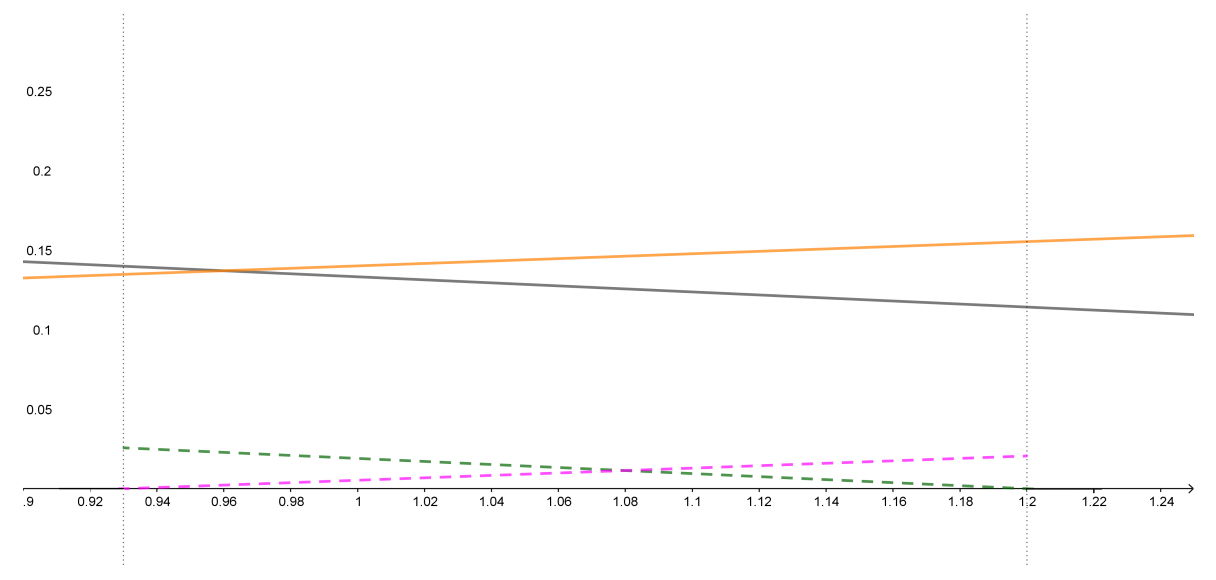

(b) Benchmark vs Lower Cost Asymmetry: Benchmark case $\left(c_{1}=0.8\right.$ and $\left.c_{2}=1.2\right)$ : Grey $=\left(x_{1}^{2}\right)^{O L}$ and Orange $=\left(x_{1}^{2}\right)^{F}$. Lower Cost Asymmetry $\left(c_{1}=0.9\right.$ and $\left.c_{2}=1.1\right)$ : Green $=\left(x_{1}^{2}\right)^{O L}$ and Magenta $=\left(x_{1}^{2}\right)^{F}$.

Figure 2: Quality differences in steady-state. 